\title{
The Establishment of Tumor Necrosis Factor Receptor-associated Protein 1 (TRAP1) Transgenic Mice and Severe Fat Accumulation in the Liver of TRAP1 Mice during Liver Regeneration
}

\author{
Chang-Nim Im ${ }^{1,2,3}$, Ying Zheng ${ }^{1,2}$, Sun Hye Kim ${ }^{1,2,4}$, Tai-Oin Huang ${ }^{1,2,5}$, Du-Hyong Cho ${ }^{6}$ and Jeong-Sun Seo ${ }^{1,2, *}$ \\ 'Department of Biochemistry and Molecular Biology, College of Medicine, Seoul National University, Seoul, Korea \\ ${ }^{2}$ ILCHUN Molecular Medicine Institute MRC, Seoul National University, Seoul, Korea \\ ${ }^{3}$ Division of Radiation Cancer Research, Korea Institute of Radiological and Medical Sciences, Seoul, Korea \\ ${ }^{4}$ Cutaneous Biology Research Center Massachusetts General Hospital, Harvard Medical School, Boston, USA \\ ${ }^{5}$ Department of Biochemistry and Biophysics, University of North Carolina, NC, USA \\ ${ }^{6}$ Department of Neurology, Konkuk University Medical Center, Konkuk University, Korea
}

Subject areas; Biological frontiers (General Biology)

Author contribution; C-N. I. designed, performed experiments and wrote manuscript. $Y$. Z. and S. H. K. performed experiments and wrote manuscript. T-O. H. wrote manuscript and D-H.

C. interpreted data and provided critical comments for this manuscript

*Correspondence and requests for materials should be addressed to J-S.S. (jeongsun@snu. ac.kr).

Editor; Keun Woo Lee, Gyeongsang National University, Korea

Received November 22, 2013

Accepted December 23, 2013

Published December 24, 2013

Citation; Im, C-N., et al. The Establishment of Tumor Necrosis Factor Receptor-associated Protein 1 (TRAP1) Transgenic Mice and Severe Fat Accumulation in the Liver of TRAP1 Mice during Liver Regeneration. IBC 2013, 5:9, 1-7. doi: 10.4051/ibc.2013.5.4.0009

Funding; Chang-Nim Im was supported by the second stage of the Brain Korea 21 Project in 2007-2008.

Competing interest; All authors declare no financial or personal conflict that could inappropriately bias their experiments or writing.

(c) Im, C-N. et al. This is an Open Access article distributed under the terms of the Creative Commons Attribution Non-Commercial License (http://creativecommons.org/licenses/by-nc/3.0) which permits unrestricted non-commercial use, distribution, and reproduction in any medium, provided the original work is properly cited.

\section{SYNOPSIS}

Introduction: Tumor necrosis factor receptor-associated protein 1 (TRAP1) is a mitochondrial heat shock protein (HSP), which belongs to HSP90 family. It plays important roles in regulating mitochondrial integrity, protecting against oxidative stress, and inhibiting cell death. Recent studies suggest that TRAP1 is linked to mitochondria and its metabolism. In this study, we established TRAP1 transgenic mice and performed partial hepatectomy (PH) on wild-type (WT) and TRAP1 transgenic mice to investigate the function of TRAP1 during liver regeneration.

Results and Discussion: We found that TRAP1 was highly expressed in liver as well as kidney. In addition, liver regeneration slightly decreased together with increased fatty liver and inflammation at $72 \mathrm{hr}$ after PH in TRAP1 transgenic mice compared with WT control group mice. Concomitantly, we observed decreased levels of p38 protein in TRAP1 transgenic mice compared with WT control group mice. These results suggest that TRAP1 plays a critical role in liver energy balance by regulating lipid accumulation during liver regeneration.

Conclusions and Prospects: To our knowledge, we reported, for the first time, that liver regeneration slightly reduced together with increased fat accumulations after PH in TRAP1 transgenic mice compared with WT control group mice. Concomitantly, we observed decreased levels of p38 protein in TRAP1 transgenic mice compared with WT control group mice. Overexpression of TRAP1 might affect liver regeneration via disturbing mitochondrial function leading to fatty liver in vivo.

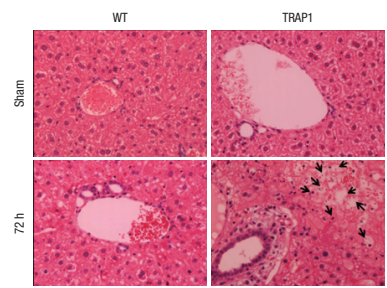

Key Words: fatty liver; mitochondria; partial hepatectomy; p38; TRAP1 


\section{INTRODUCTION}

Mitochondria are distinct cellular organelles composed of a double-membrane system. Moreover mitochondria are vitally important organelles, in which the majority of the ATP is synthesized via oxidative phosphorylation. Tumor necrosis factorassociated protein 1 (TRAP1) is a mitochondrial heat shock protein (HSP) belonging to HSP90 family ${ }^{1}$ and its expression was also elevated in many cancers. TRAP1 plays important roles in regulating mitochondrial integrity, protecting against oxidative stress, and inhibiting cell death ${ }^{2}$. TRAP1 protects cells from ROS-induced apoptosis and senescence ${ }^{3-6}$. In addition, Takamura et al. reported that stable TRAP1 knockdown cells showed abnormal mitochondrial morphology, as well as significant decreases in dynamin-related protein 1 and mitochondrial fission proteins $^{7}$. Zhang et al. reported that TRAP1 protects against cardiac hypertrophy and fibrosis using in vivo mice model ${ }^{8}$. Recent studies demonstrate that TRAP1 is linked to mitochondria and its metabolism and it regulates a metabolic switch between mitochondrial respiration and aerobic glycolysis ${ }^{9}$. These evidences suggest that TRAP1 may play a role in mitochondrial integrity and metabolism.

The liver is one of vital organs in vertebrates and some other animals. Mitochondria are abundant in liver. Moreover, mitochondria are the main energy source in hepatocytes and play a major role in extensive oxidative metabolism and normal function of the liver ${ }^{10}$. Liver is the only human internal organ capable of natural regeneration of lost tissue; as little as $30 \%$ of a liver can regenerate into a whole liver. Liver regeneration after partial hepatectomy $(\mathrm{PH})$ is a perfectly orchestrated process of compensatory growth that proceeds in stages ${ }^{11}$. The main event of liver regeneration is hepatocyte replication followed by mitosis ${ }^{12}$.

TRAP1 mRNA and protein are highly expressed in cancer cell lines and tumors ${ }^{13,14}$, however, little is known about the effect of TRAP1 overexpression on mitochondria after PH conditions. We recently investigated the effect of mitochondrial TRAP1 overexpression in a mouse fibroblast cell line, NIH/3T3 and found that overexpression of TRAP1 resulted in a series of mitochondrial aberrations ${ }^{15}$. In order to study the role of TRAP1 in vivo, we established TRAP1 transgenic mice and found that TRAP1 overexpression was predominant in liver tissue. To further elucidate the role of TRAP1 on liver, we investigated the effect of mitochondrial TRAP1 overexpression on liver regeneration under $\mathrm{PH}$ conditions.

\section{RESULTS}

\section{Establishment of TRAP1 transgenic mice}

We established TRAP1 transgenic mice as described in Materials and Methods. To confirm that TRAP1 vector was inserted in transgenic mice, we performed genomic DNA PCR analysis. As shown in Figure 1A, specific products for TRAP1 appeared only in TRAP1 transgenic mice both two lines. It has been known that TRAP1 is a mitochondrial protein. Therefore, we determined whether overexpression of TRAP1 was targeted to mitochondria via subcellular fractionation in livers from wild type and TRAP1
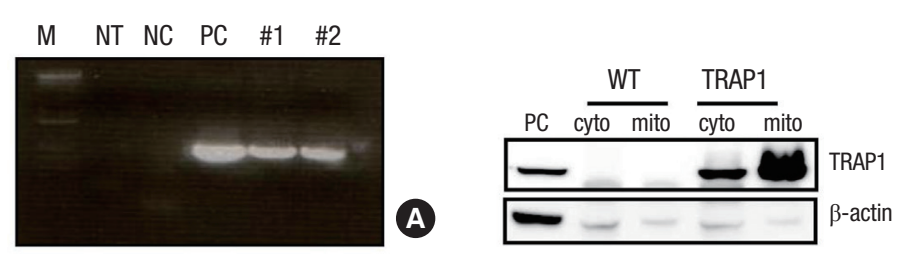

B
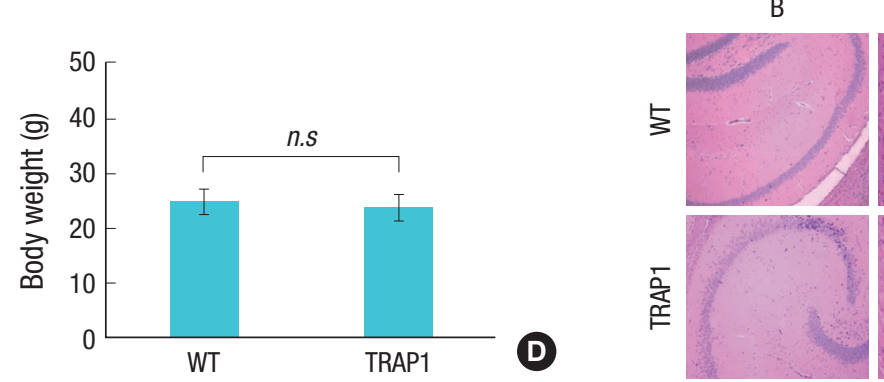

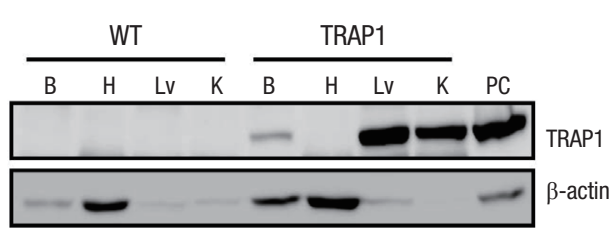

C

Figure 1. Establishment of TRAP1 transgenic mice. (A) Genomic DNA PCR against TRAP1 in wild type and TRAP1 transgenic mice. NT: no template, NC: normal mouse genomic DNA, PC: positive control and TRAP1 transgenic mice (\#1 and \#2: transgenic mouse line number). (B) TRAP1 expression in mitochondrial fraction. Tissues were fractionated into cytosolic (cyto) and mitochondrial (mito) fractions, and subjected to Western blotting against TRAP1. (C) TRAP1 expression in wild type (WT) and TRAP1 transgenic mice tissues. Protein levels were analyzed via Western blotting using specific antibody for human TRAP1. (B; brain, H; heart, LV; liver, K; kidney, PC; positive control). (D) Measurement of Body weight in WT and TRAP1 transgenic mice $(n=5)$. (E) Morphology of tissues of WT and TRAP1 transgenic mouse. Representative organs were dissected from mice and fixed. Sections were obtained from paraffin-embedded tissue samples, stained with hematoxylin and eosin (H\&E), and examined under a microscope and photographed. (B: brain; × 100, H: heart, Lv: liver, and K: kidney, $\times 200$ ) 
transgenic mice respectively via Western blot analysis using human specific TRAP1 antibody. In a previous report ${ }^{4}$, we observed that $\beta$-actin could be utilized as a cytosolic marker compared with a mitochondrial marker. Therefore, $\beta$-actin was used as a cytoplasmic marker protein in Figure 1B. Although small amount of TRAP1 was detected in cytosolic fraction, most TRAP1 was found in mitochondria. We assessed the tissue distribution of TRAP1 in these mice. Brain, heart, liver and kidney tissues from WT and TRAP1 transgenic mice were lysed and Western blot analysis against TRAP1 was performed in those tissues as described in Materials and Methods. As shown in Figure 1C, TRAP1 was significantly overexpressed in liver and kidney of TRAP1 transgenic mice. Next, we measured body weight under physiological condition in WT and TRAP1 transgenic mice. Figure 1D shows that there was no significant difference between two types of mice group. We also dissected representative tissues including liver from WT or transgenic mice and performed hematoxylin and eosin (H\&E) staining. As shown in Figure 1E, there was no significant difference between WT and TRAP1 transgenic mice. These data suggested that TRAP1 transgenic mice were successfully generated and their physiological conditions were not impact by overexpression of TRAP1 although these analyses were limited in some tissues.

\section{Liver regeneration in WT and TRAP1 transgenic mice after PH}

Since TRAP1 was highly expressed in liver, which is rich in mitochondria, we utilized partial hepatectomy $(\mathrm{PH})$ model system to evaluate whether overexpression of TRAP1 affects liver re- generation. After operation with or without two-thirds $\mathrm{PH}$ as mentioned in Materials and Methods, we measured body weight and liver weight in WT and TRAP1 transgenic mice. There was no significant difference in body weight between WT and TRAP1 transgenic mice $72 \mathrm{hr}$ after sham- or PH-operated groups (Figure 2A; upper panel). Difference in liver weight also was not significant either between WT and TRAP1 transgenic mice 72 hr after sham operation (Figure 2A; lower left panel). However, we found that liver weight was significantly decreased in $\mathrm{PH}-$ operated TRAP1 transgenic mice compared with PH-operated wild type mice $72 \mathrm{hr}$ after operation (Figure 2A; lower right panel, $P=0.044$ ). Figure $2 \mathrm{~B}$ shows the ratio of liver weight to body weight in WT and TRAP1 mice at the time point of 24, 48, and $72 \mathrm{hr}$ after $\mathrm{PH}(P=0.062)$. Interestingly, as shown in Figure 3 , TRAP1 transgenic mice developed severe fatty livers $48 \mathrm{hr}$ (60\%; three of five mice) and $72 \mathrm{hr}$ ( $40 \%$; two of five mice) after $\mathrm{PH}$ but not in WT control mice ( $0 \%$; zero of five mice). These results suggest that overexpression of TRAP1 decreased in vivo liver regeneration in response to $\mathrm{PH}$.

\section{Pathological and morphological analysis of WT and} TRAP1-overexpressed liver tissues after 72 hr of PH Next, we analyzed liver tissues in WT and TRAP1 transgenic mice after PH. Livers were dissected from WT mice and TRAP1 transgenic mice $72 \mathrm{hr}$ after sham operation or $\mathrm{PH}$, respectively and fixed in $10 \%$ formalin. Sections were obtained from paraffin-embedded tissue samples, stained with $\mathrm{H} \& \mathrm{E}$, and examined under a microscope. Figure $3 \mathrm{~A}$ shows that there was an increase of fat accumulation in TRAP1 transgenic mice compared
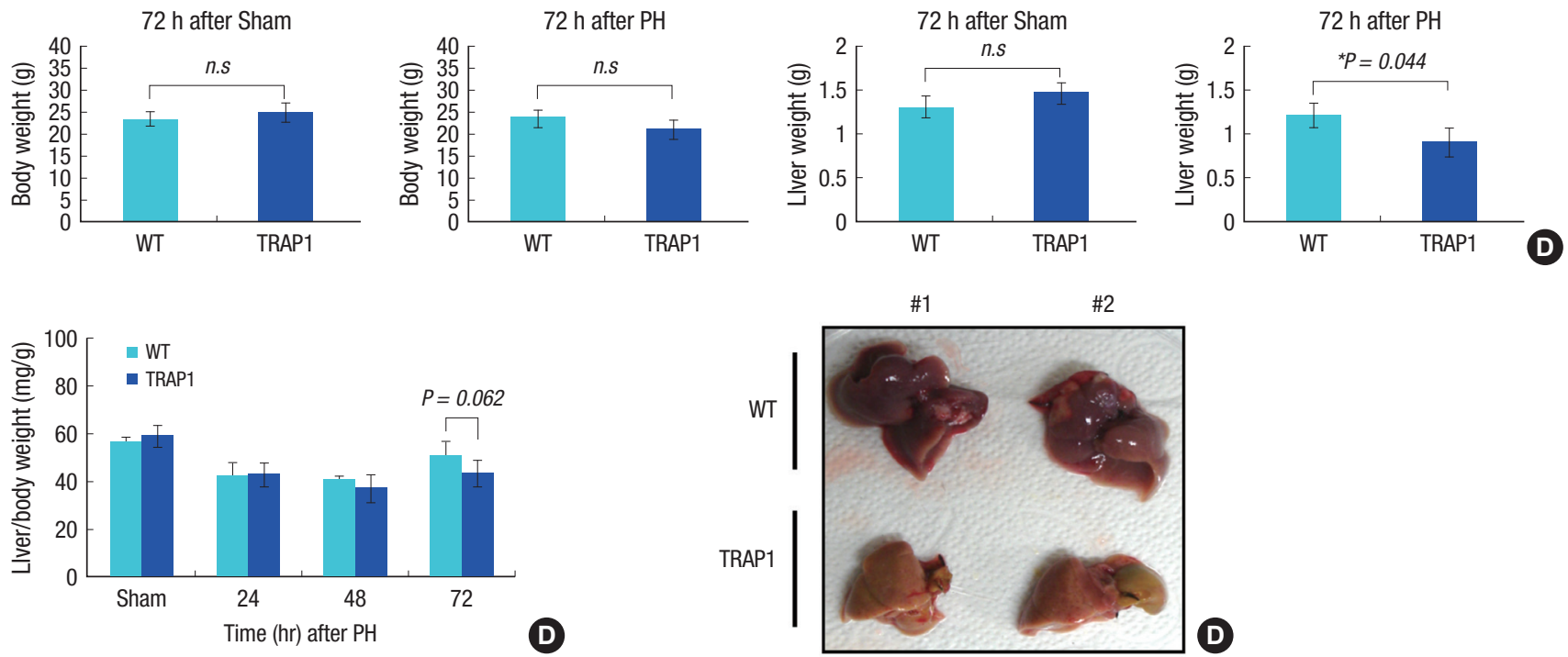

Figure 2. Liver regeneration in WT and TRAP1 transgenic mice after PH. (A) Body weight and liver weight at the time point of $72 \mathrm{hr}$ after sham or PH in WT and TRAP1 transgenic mice. (B) Liver weight per body weight was calculated after $24 \mathrm{hr}, 48 \mathrm{hr}$, and $72 \mathrm{hr}$ after PH in wild type and TRAP transgenic mice, respectively $(n=3-5)$. Sham-operated mice were treated without liver resection. (C) Fat accumulations in liver after $72 \mathrm{hr}$ of PH. Livers were dissected from mice after $72 \mathrm{hr}$ of PH in wild type (WT) and TRAP1 transgenic mice. Representative livers were photographed. 

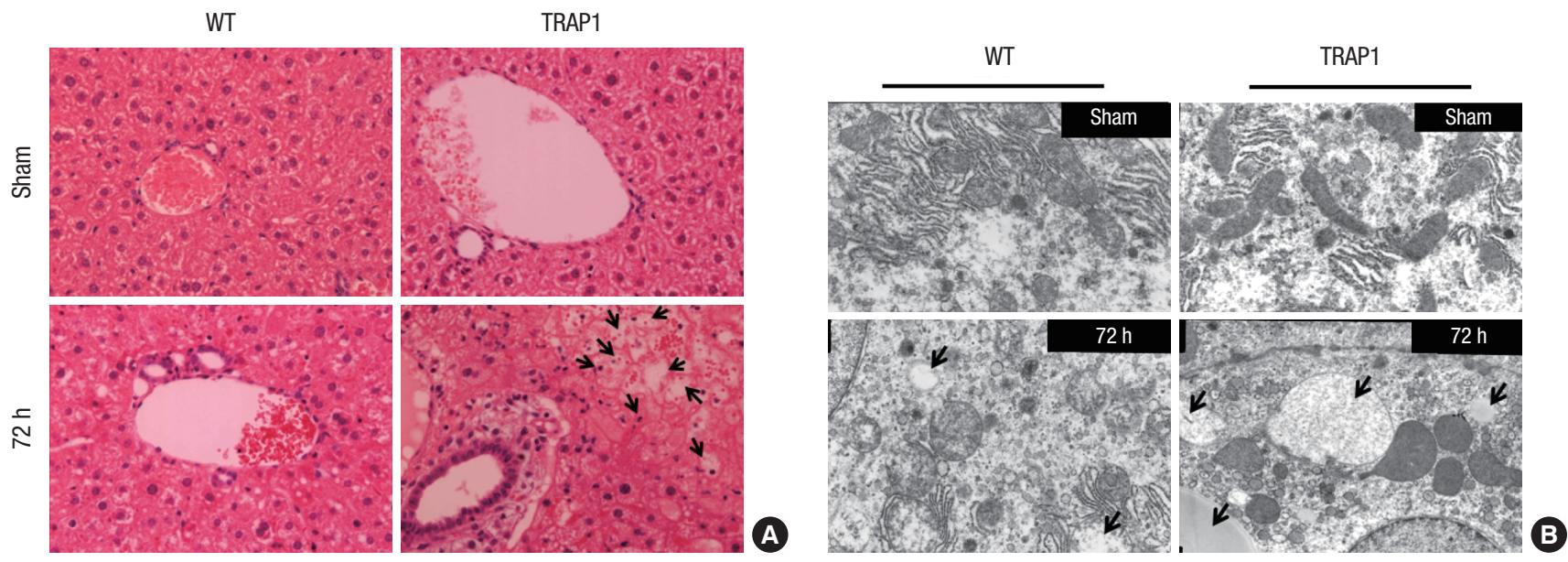

Figure 3. Pathological analysis of liver tissues after $72 \mathrm{hr}$ of PH in wild type (WT) and TRAP1 transgenic mice. (A) Hematoxylin and eosin (H\&E) staining. Livers were dissected from mice and fixed. Sections were obtained from paraffin-embedded tissue samples, stained with H\&E, and examined under a microscope and photographed ( $\times 400)$. (B) Electron microscopy. Liver tissues were fixed as described in Materials and Methods. Samples were visualized under electron microscope $(\times$ 10,000). Arrows indicate lipid accumulation.
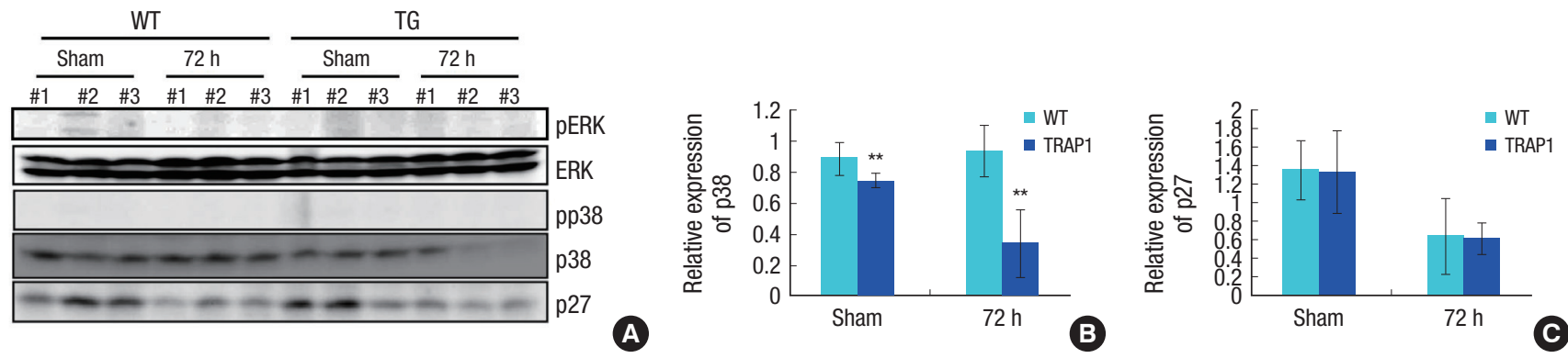

Figure 4. Western blot analysis of livers after $72 \mathrm{hr}$ of PH in wild type (WT) and TRAP1 transgenic mice. (A) Proteins from liver tissues were extracted, and subjected to Western blotting against specific antibodies. (B) and (C) Intensities of images from (A) was measured using Image $\mathrm{J}$ program, normalized by ERK and represented as relative expression to sham group of wild type mice. ${ }^{*} P<0.05$, ${ }^{\star \star} P<0.01$ vs. wild type mice

with WT control mice $72 \mathrm{hr}$ after PH. Since TRAP1 is a mitochondrial protein and mitochondria are abundant in liver, we examined TRAP1-induced morphological change of mitochondria via electron microscope. As described in Materials and Methods, liver tissues were fixed and visualized under electron microscope. We could not detect significant morphological alterations in mitochondria between WT and TRAP1 mice during liver regeneration. Instead, we found increased lipid droplets in TRAP1 transgenic mice compared with WT control mice $72 \mathrm{hr}$ after PH similar to H\&E staining result (Figure 3B).

\section{Expression levels of p27 and p38 in WT and TRAP1-}

overexpressed liver tissues $72 \mathrm{hr}$ after PH

Since MAPK such as $\mathrm{p} 38^{16}$ and ERK and $\mathrm{p} 27^{17}$ participates in cell cycle regulation, and function as a suppressor of cell proliferation during $\mathrm{PH}$, we determined phosphorylation of p38 and ERK in WT control mice and TRAP1 transgenic mice during liver regeneration after sham-operation or $\mathrm{PH}$. Figure $4 \mathrm{~A}$ shows that phosphorylation of p38 and ERK was not detected both in
WT control mice and TRAP1 transgenic mice $2 \mathrm{hr}$ after PH. However, total p38 protein levels were decreased in TRAP1 transgenic mice $72 \mathrm{hr}$ after PH. There was no significant difference in total ERK protein levels between WT and TRAP transgenic mice. We also examined expression levels of p27 under the same condition. Moreover, expression of p27 protein decreased both in WT and TRAP1 transgenic mice $72 \mathrm{hr}$ after PH compared with sham-operation; however, there was no difference between WT and TRAP1 transgenic mice.

\section{DISCUSSION}

It has been shown that TRAP1, the mitochondrial Hsp90 homolog, is highly expressed in many cancers. It plays many important roles in regulating mitochondrial integrity, protecting against oxidative stress, and inhibiting cell death ${ }^{2}$. In addition, stable TRAP1 knockdown cells showed abnormal mitochondrial morphology, as well as significant decreases in mitochondrial fission proteins. These results suggest that TRAP1 regulates the 
expression of fission proteins and affects mitochondrial function $^{7}$. A recent report showed that TRAP1 protects against cardiac hypertrophy and fibrosis in vivo mice model ${ }^{8}$. However, it remains unknown about the effects of TRAP1 overexpression in vivo. In the present study, we generated TRAP1 transgenic mice and investigated the function of TRAP1 in vivo during liver regeneration. We observed the differential tissue distribution of TRAP1 expression in TRAP1 transgenic mice. To drive ectopic gene expression in various in vivo contexts, we utilized a constitutive promoter of elongation factor- 1 alpha which consists of two isoforms eEF1A1 and eEF1A2. In mammalian, eEF1A1 is ubiquitously expressed, whereas eEF1A2 expression is switchedon in adult life in specialized tissues such as skeletal muscle, cardiomyocytes and nervous system ${ }^{18}$. Hence, we speculate that protein expression level varies in mice unlike in the cell lines and TRAP1 expression levels varied in tissues of transgenic mice.

We demonstrated that liver regeneration slightly reduced together with increased fat accumulation and inflammation at 72 hr after PH in TRAPl transgenic mice compared with WT control group mice. Concomitantly, we observed decreased p38 protein levels in TRAP1 transgenic mice compared with WT control group mice.

It has been reported that p38 participates in cell cycle regulation, functioning as a suppressor of cell proliferation and tumorigenesis. Campbell et al. reported that p38 is activated in normal liver, however, it is rapidly inactivated starting 30 minutes after $\mathrm{PH}$, and is re-activated by $12 \mathrm{hr}^{16}$. In the present study, we observed that p38 protein levels were significantly decrease of in TRAP1 mice at $72 \mathrm{hr}$ after PH compared with WT mice group. However, we could not observe activation of p38 at 24, 48, and $72 \mathrm{hr}$ after PH in both WT and TRAP1 mice groups. We speculate a possibility that we examined activation of $\mathrm{p} 38$ at late time after $\mathrm{PH}$. Yu et al., recently suggested the complicated modulation of fatty acid synthase (FASN) degradation by concerted action of p38, E3 ligase, and Shp2 $2^{19}$. They reported that $\mathrm{p} 38$ is activated by fasting and it stimulates FASN protein degradation in mice. Considering that p38 protein level decreased after PH in TRAP1 transgenic mice together with fatty liver, it suggested that there is a link between $\mathrm{p} 38$ and fatty liver during PH in TRAP1 transgenic mice, which requires further study. Besides p38, p27 has also been involved in regulating re-entry of residual hepatocytes into the cell cycle upon loss of liver tissue by PH. Rohlfing et al. reported that $\mathrm{PH}$ in rats results in immediate down-regulation of p27 in residual liver tissue by transcriptional and post-translational processes ${ }^{17}$. Similarly, we also observed that $\mathrm{p} 27$ protein levels were reduced in both WT and TRAP1 mouse at $72 \mathrm{hr}$ after $\mathrm{PH}$.

Interestingly and unexpectedly, we found that liver regeneration was slightly decreased together with increased fat accumulation and inflammation at $72 \mathrm{hr}$ after PH in TRAP1 transgenic
Table 1. Summary of fatty liver incidence after PH.

\begin{tabular}{lcccc}
\hline & Sham & $24 \mathrm{~h}$ & $48 \mathrm{~h}$ & $72 \mathrm{~h}$ \\
\hline WT & $0 / 5$ & $0 / 5$ & $0 / 5$ & $0 / 5$ \\
TRAP1 & $0 / 5$ & $0 / 5$ & $3 / 5$ & $2 / 5$ \\
\hline
\end{tabular}

Number of mice with fatty liver were counted at the time point of 24,48 , and $72 \mathrm{hr}$ af ter sham or PH in WT and TRAP1 transgenic mice $(n=5)$.

mice compared with WT control group mice. The incidence of fatty liver in TRAP1 transgenic mice was significantly higher (about 50\%) than that of WT control mice ( $0 \%$ ) at 48 and $72 \mathrm{hr}$ after PH (Table 1). We also observed increased fat accumulation via electron microscopy in TRAP1 mice at $72 \mathrm{~h}$ after PH. Fatty liver is a condition that large vacuoles of triglyceride fat accumulate in liver cells via the process of steatosis. It has been shown that the most common liver disease, affecting one-third of the overall population, is nonalcoholic fatty liver disease (NAFLD), which is characterized by an intrahepatic accumulation of lipids. NAFLD can evolve into nonalcoholic steatohepatitis (NASH) in the presence of oxidative stress and inflammation. Moreover, NASH is a serious risk factor for disabling and deadly liver diseases such as cirrhosis and hepatocellular carcinoma ${ }^{20}$. Colak et al. reported distinct molecular mechanisms for early hepatocellular carcinoma (HCC) and they developed a rat model of liver regeneration post-hepatectomy, as well as liver cells undergoing malignant transformation and compared them to normal liver using a microarray approach and observed that over $70 \%$ of signature genes including p53 and p38 were cancer-related ${ }^{21}$. It has been reported altered mitochondrial functions in a variety of chronic liver diseases including alcohol-induced liver disease, nonalcoholic fatty liver disease, viral hepatitis, and cholestasis ${ }^{10}$.

A recent study suggests that TRAP1 regulates a metabolic switch between mitochondrial respiration and aerobic glycolysis as well as mitochondrial fusion/fission. TRAP1-deficiency promotes an increase in mitochondrial respiration and fatty acid oxidation and reactive oxygen species together with suppression of glucose metabolism ${ }^{9}$. Moreover, recent our report suggests that overexpression of TRAP1 leads to aberrations of mitochondria $^{15}$. Considered that TRAP1 is an important regulator of not only mitochondrial structure but also metabolism, it is possible to speculate from our findings that overexpression of TRAP1 has effects on mitochondria and a series of metabolic process such as fatty acid oxidation leading to fatty liver during liver regeneration after PH in vivo. It requires further studies to elucidate the mechanism of fatty liver in TRAP1 transgenic mice after PH and the expression of TRAP1 in human fatty liver samples.

\section{CONCLUSION AND PROSPECTS}

In summary, we investigated the function of TRAP1 during liver 
regeneration after PH on WT and TRAP1 transgenic mice. To our knowledge, we reported, for the first time, that liver regeneration slightly reduced together with increased fat accumulations after PH in TRAP1 transgenic mice compared with WT control group mice. Concomitantly, we observed decreased levels of p38 protein in TRAP1 transgenic mice compared with WT control group mice. These results suggest that TRAP1 plays a critical role in liver energy balance by regulating lipid accumulation during liver regeneration. Overexpression of TRAP1 might affect liver regeneration via disturbing mitochondrial function leading to fatty liver in vivo.

\section{MATERIALS AND METHODS}

\section{Vector preparation}

pcDNA3.1-TRAP1 plasmid containing human full length TRAP1 was prepared via subcloning into the pcDNA3.1 vector, under the control of the EFl $\alpha$-promoter (Macrogen, Inc., Korea) with the original pOTB7-TRAP1 vector (the Korea Research Institute of Bioscience and Biotechnology, Korea).

\section{Production of human TRAP1 transgenic mice}

Standard microinjection procedures were used for transgenic mice production (Macrogen, Inc., Korea). Briefly, fertilized mouse eggs were flushed from the oviducts of superovulated C57BL/6NCrjBgi mice, and male pronuclei were injected with pcDNA3.1-TRAP1 $(2 \mathrm{ng} / \mu \mathrm{L})$ that had been linearized with the PI SceI enzyme at its cognate restriction site located in the pcDNA3.1 vector. The injected eggs were reimplanted in the oviducts of pseudo-pregnant ICR recipient females. At 3 weeks of age, the animals were tested for the presence of the transgene by PCR analysis. $100 \mu \mathrm{g}$ of genomic DNA was amplified via PCR, as follows: denaturation for 5 minutes at $94^{\circ} \mathrm{C}, 35$ cycles of denaturation for 30 seconds at $94^{\circ} \mathrm{C}, 30$ seconds of annealing at $60^{\circ} \mathrm{C}$, and 30 seconds of extension at $72^{\circ} \mathrm{C}$, and a final extension for 10 minutes at $72^{\circ} \mathrm{C}$, using specific primers spanning human TRAP1 and EF-1 $\alpha$ promoter region (sense: 5 ' - gggagctcaaaatggaggac $-3^{\prime}$ and antisense: $5^{\prime}$ - gatggtgatggtgctttct $\left.-3^{\prime}\right)$. The specific PCR products were resolved on $1 \%$ agarose gels, and visualized via ethidium bromide staining.

\section{Animals}

We obtained the approval for all animal experiments from Seoul National University Council (SNU-070328-1). Mice (C5BL/6N) were housed in cages (in groups of 5 mice) with food and water freely available and under a standard specific pathogen free (SPF) zone. Humidity $(50 \pm 10 \%)$ and temperature $\left(22 \pm 2^{\circ} \mathrm{C}\right)$ were kept constant, and a $12 \mathrm{hr}$ light/dark cycle was used (lights on, 7:00 a.m. to 7:00 p.m.). All tests were conducted during the light cycle, and the procedures were per- formed in accordance with guidelines.

\section{Partial hepatectomy}

Partial hepatectomy (PH) was performed on 6- to 8-week-old male TRAP $1^{+-}$and their wild type TRAPl ${ }^{-/-}$littermates under anesthesia using the mixture of zoletil 50 and rompun ${ }^{22}$. Mice $(n=5)$ were sacrificed at different time points and the liver remnants were removed, weighed, and frozen or processed for molecular and histological experiments, respectively. Shamoperated mice were treated as above without liver resection.

\section{Western blotting}

Tissues were lysed in a buffer containing $50 \mathrm{mM}$ Tris, $\mathrm{pH}$ 7.4, $150 \mathrm{mM} \mathrm{NaCl}, 1 \mathrm{mM}$ EDTA, $1 \%$ sodium dodecyl sulphate (SDS), complete protease inhibitor cocktail, $1 \mathrm{mM}$ phenylmethylsulfonyl fluoride, and $1 \mathrm{mM} \mathrm{NaF}$, for 30 minutes on ice. After homogenizing, lysates were centrifuged, quantitated with protein assay reagent (Bio Rad), and subjected to sodium dodecyl sulphate-polyacrylamide gel electrophoresis (SDS-PAGE). The proteins were transferred to nitrocellulose membranes (S\&S). The membranes were blocked with $5 \%$ (w/v) nonfat dry milk in $1 \times$ Tris buffered saline containing $0.1 \%$ Tween- 20 (TBS-T), for $1 \mathrm{hr}$ at room temperature. Each of the proteins was detected with its specific antibody (anti-mouse TRAP1 antibody from NeoMarkers, anti-mouse $\beta$ - actin and ERK antibodies from Santa Cruz Biotechnology and anti-rabbit phospho-ERK (Thr202/ Tyr204) antibody, anti-rabbit phospho-p38 and p38 antibodies, anti-rabbit p27 antibody from Signaling). The antibody-antigen complex was detected with horseradish peroxidase-conjugated secondary antibody (Santa Cruz Biotechnology), and visualized via the standard chemiluminescence technique, which was conducted in accordance with the manufacturer's provided instructions (Pierce). Images were obtained on LAS 3000 (Fujifilm).

\section{Subcellular fractionation}

The cytosolic and the mitochondrial fractions were isolated with digitonin (Sigma). In brief, tissues were lysed in lysis buffer (80 $\mathrm{mM} \mathrm{KCl}, 250 \mathrm{mM}$ sucrose, $200 \mu \mathrm{g} / \mathrm{mL}$ digitonin in PBS) for 5 minutes on ice. After 5 minutes of centrifugation at $10,000 \mathrm{~g}$, the supernatant was used as a cytosolic fraction. The pellets were resuspended in buffer containing $50 \mathrm{mM}$ Tris- $\mathrm{Cl}$ (pH 7.5), 150 mM NaCl, 2 mM EDTA, 2 mM EGTA, 0.2\% TritonX-100, and $0.3 \%$ NP- 40 , and incubated for 10 minutes on ice. The supernatants were used as a mitochondrial fraction after 5 minutes of centrifugation at 10,000 g. The samples of each fraction were then quantitated using protein assay reagent (Bio-Rad).

Hematoxylin and eosin staining

Various organs were dissected from mice and fixed in $10 \%$ formalin. Sections were obtained from paraffin-embedded tissue 
samples, stained with hematoxylin and eosin (H\&E), and examined under a microscope and photographed. Pathological analysis was carried out in the Department of Pathology Seoul National University.

\section{Electron microscopy}

Liver tissues were fixed with $2.5 \%$ glutaraldehyde, and post-fixed in $1 \%$ buffered osmium tetroxide. After dehydration with a graded ethanol series and embedding in Epoxy resin (Poly bed 812 kit), ultra-thin sections were prepared using an ultra-microtome (RMC products-MTXL) equipped with a diamond knife. Heavy metal staining was conducted with $4 \%$ uranyl acetate and lead citrate solutions, and samples were visualized under electron microscope (Tokyo, Japan) at a magnification $(\times 10,000)$.

\section{Statistical Analysis}

Student's t-tests were employed in order to compare the differences between two different groups. $P$ value of $<0.05$ was considered statistically significant.

\section{ACKNOWLEDGEMENTS}

Chang-Nim Im was supported by the second stage of the Brain Korea 21 Project in 2007-2008.

\section{REFERENCES}

1. Felts, S. J., Owen, B. A., Nguyen, P., Trepel, J., Donner, D. B., and Toft, D. O. (2000). The hsp90-related protein TRAP1 is a mitochondrial protein with distinct functional properties. The Journal of Biological Chemistry 275, 3305-3312.

2. Kang, B. H. (2012). TRAP1 regulation of mitochondrial life or death decision in cancer cells and mitochondria-targeted TRAP1 inhibitors. BMB Reports 45, 1-6.

3. Hua, G., Zhang, Q., and Fan, Z. (2007). Heat shock protein 75 (TRAP1) antagonizes reactive oxygen species generation and protects cells from granzyme M-mediated apoptosis. The Journal of Biological Chemistry 282, 20553-20560.

4. Im, C. N., Lee, J. S., Zheng, Y., and Seo, J. S. (2007). Iron chelation study in a normal human hepatocyte cell line suggests that tumor necrosis factor receptor-associated protein 1 (TRAP1) regulates production of reactive oxygen species. Journal of Cellular Biochemistry 100, 474-486.

5. Masuda, Y., Shima, G., Aiuchi, T., Horie, M., Hori, K., Nakajo, S., Kajimoto, S., Shibayama-Imazu, T., and Nakaya, K. (2004). Involvement of tumor necrosis factor receptor-associated protein 1 (TRAP1) in apoptosis induced by beta-hydroxyisovalerylshikonin. The Journal of Biological Chemistry 279, 42503-42515.

6. Pridgeon, J. W., Olzmann, J. A., Chin, L. S., and Li, L. (2007). PINK1 protects against oxidative stress by phosphorylating mitochondrial chaperone TRAP1. PLoS Biology 5, el72.

7. Takamura, H., Koyama, Y., Matsuzaki, S., Yamada, K., Hattori, T., Miya- ta, S., Takemoto, K., Tohyama, M., and Katayama, T. (2012). TRAP1 controls mitochondrial fusion/fission balance through Drp1 and Mff expression. PloS one 7, e51912.

8. Zhang, Y., Jiang, D. S., Yan, L., Cheng, K. J., Bian, Z. Y., and Lin, G. S. (2011). HSP75 protects against cardiac hypertrophy and fibrosis. Journal of Cellular Biochemistry 112, 1787-1794.

9. Yoshida, S., Tsutsumi, S., Muhlebach, G., Sourbier, C., Lee, M. J., Lee, S., Vartholomaiou, E., Tatokoro, M., Beebe, K., Miyajima, N., et al. (2013). Molecular chaperone TRAP1 regulates a metabolic switch between mitochondrial respiration and aerobic glycolysis. Proceedings of the National Academy of Sciences of the United States of America 110, E1604-1612.

10. Grattagliano, I., Russmann, S., Diogo, C., Bonfrate, L., Oliveira, P. J., Wang, D. Q., and Portincasa, P. (2011). Mitochondria in chronic liver disease. Current Drug Targets 12, 879-893.

11. Taub, R. (2004). Liver regeneration: from myth to mechanism. Nature reviews Molecular Cell Biology 5, 836-847.

12. Fausto, N., Campbell, J. S., and Riehle, K. J. (2006). Liver regeneration. Hepatology 43, S45-53.

13. Kang, B. H., Plescia, J., Dohi, T., Rosa, J., Doxsey, S. J., and Altieri, D. C. (2007). Regulation of tumor cell mitochondrial homeostasis by an organelle-specific Hsp90 chaperone network. Cell 131, 257-270.

14. Song, H. Y., Dunbar, J. D., Zhang, Y. X., Guo, D., and Donner, D. B. (1995). Identification of a protein with homology to hsp90 that binds the type 1 tumor necrosis factor receptor. The Journal of Biological Chemistry 270, 3574-3581.

15. Im, C. N., and Seo, J. S. (2013). Overexpression of tumor necrosis factor receptor-associated protein 1 (TRAP1), leads to mitochondrial aberrations in mouse fibroblast NIH/3T3 cells. BMB Reports.

16. Grisham, J. W. (1962). A morphologic study of deoxyribonucleic acid synthesis and cell proliferation in regenerating rat liver; autoradiography with thymidine-H3. Cancer Research 22, 842-849.

17. Campbell, J. S., Argast, G. M., Yuen, S. Y., Hayes, B., and Fausto, N. (2011). Inactivation of p38 MAPK during liver regeneration. The International Journal of Biochemistry \& Cell Biology 43, 180-188.

18. Colak, D., Chishti, M. A., Al-Bakheet, A. B., Al-Qahtani, A., Shoukri, M. M., Goyns, M. H., Ozand, P. T., Quackenbush, J., Park, B. H., and Kaya, N. (2010). Integrative and comparative genomics analysis of early hepatocellular carcinoma differentiated from liver regeneration in young and old. Molecular Cancer 9, 146.

19. Rohlfing, A. K., Trescher, K., Hahnel, J., Muller, C., and Hildebrandt, J. P. (2013). Partial hepatectomy in rats results in immediate down-regulation of p27Kip1 in residual liver tissue by transcriptional and posttranslational processes. Frontiers in Physiology 4, 139.

20. Yang, S., Leow, C. K., and Tan, T. M. (2006). Expression patterns of cytokine, growth factor and cell cycle-related genes after partial hepatectomy in rats with thioacetamide-induced cirrhosis. World Journal of Gastroenterology: WJG 12, 1063-1070.

21. Seo, J. S., Park, Y. M., Kim, J. I., Shim, E. H., Kim, C. W., Jang, J. J., Kim, S. H., and Lee, W. H. (1996). T cell lymphoma in transgenic mice expressing the human Hsp70 gene. Biochemical and Biophysical Research Communications 218, 582-587.

22. Higgins, G. M. P. J. T. (1931) Experimental pathology of the liver I. Archives of Pathology. pp. 186-202. 